\title{
Passive ligament stability in natural knee: a cadaveric biomechanical study Thomas Paszicsnyek ${ }^{1}$, Edoardo Bori ${ }^{2}$, Bernardo Innocenti ${ }^{2}$ \\ ${ }^{1}$ Privatklinik Graz Ragnitz, Austria \\ ${ }^{2}$ BEAMS Department, ULB, Universite de Bruxelles tp@ortho-unfall.at, edoardo.bori@gmail.com, bernardo.innocenti@ulb.ac.be
}

\section{Objectives}

Applying the correct amount of collateral ligaments tension in the knees during surgery is a prerequisite to restore normal kinematics, stability and therefore performance after TKA. In detail, a lower values of ligament tension could lead to joint instability while higher values could induce soft-tissue over-tensioning and therefore problems at later follow-up as pain and limited motion: a "functional stability" must then be defined and achieved during surgery to guarantee the best functional outcome.

In this study, an experimental ex-vivo cadaveric activity was performed to determine the minimum tension required to achieve knee functional stability.

\section{Methods}

Ten (10) fresh-frozen native knee specimens were investigated. Medical records of the donors showed that they had no history of musculoskeletal problems at the investigated knee joint. Each knee joint specimen had intact ligaments prior the test.

The femur and tibia were fixed with polyurethane foam and pegs in specific designed 3D printed fixtures and clamped to a loading frame. The test consists in applying a traction force to the femur, with a constant displacement rate and the relative force was measured by a loading-frame machine up to the stability of the joint, determined by a decrease in the derivate of the force/displacement trend followed by a plateau.

The force span between the slack region and the plateau was considered as the tension required to stabilize the joint.

This methodology was applied for joints with intact cruciate ligaments, after ACL resection and after further PCL resection, to simulate the knee behavior in native condition and prior a CR and a PS implant. 
The test was performed at $0,30,60$ and $90^{\circ}$ of flexion. Each configuration was analyzed three times for the sake of repeatability.

The experimental set-up is illustrated in figure 1 and figure 2 .

\section{Results and Discussion}

Analyzing the results for the native knee, at different flexion angles, it is possible to check that a force of $36.7 \mathrm{~N}(\mathrm{SD}=4.0 \mathrm{~N})$ is able to guarantee stability at $0^{\circ}$; similar values, with a slight higher variability, were also found for $30^{\circ}(36.7 \mathrm{~N}, \mathrm{SD}=5.7 \mathrm{~N})$ and $90^{\circ}(38.1 \mathrm{~N} \mathrm{SD}=15.4 \mathrm{~N})$. At $60^{\circ}$ a slightly statistically significantly higher tension was required $(43.3 \mathrm{SD}=5.0 \mathrm{~N})$.

The results found showed that in average a total tension of $50 \mathrm{~N}$ is sufficient to guarantee functional stability in most of the different configurations; these relatively-low tension values are in agreement with recently published studies, and the higher tensions encountered at $60^{\circ}$ of flexion are furthermore in agreement with the mid-flexion instability problem, thus showing that the results obtained can be considered reliable.

Analyzing instead the effect of different ligament configurations, in full extension, the results showed that native knee required a force of $36.7 \mathrm{~N}(\mathrm{SD}=4.0 \mathrm{~N})$, similar to the one required by an ACL resected configuration knee $(36.1 \mathrm{~N}, \mathrm{SD} 10.5 \mathrm{~N})$. When the PCL was also resected, the minimum force statistically significantly rose to a value of $46.0 \mathrm{~N}(\mathrm{SD}=10.8 \mathrm{~N})$.

The results at $0^{\circ}$ flexion for native and ACL removed specimens appears to be similar, and this may be traced back to the fact that, in full extension, ACL effects on the joint are negligible and so they do not alter excessively the stability force required, if not in terms of variability. Removing also the PCL, instead, led to a variation in needed tension also at this flexion angle since in this configuration all the stability must be provided by the collateral ligaments.

The results are in agreement to the ones found by other recent experimental (Manning, Blain, Longstaff, \& Deehan, 2018) and shown that the tension necessary to stabilize a knee joint in different ligament conditions is way lower than the ones usually applied via tensioners nowadays.

\section{Conclusion}

To reach functional stability, surgeons should consider such results intraoperatively to avoid laxity, mid-flexion instability or ligament over-tension. 


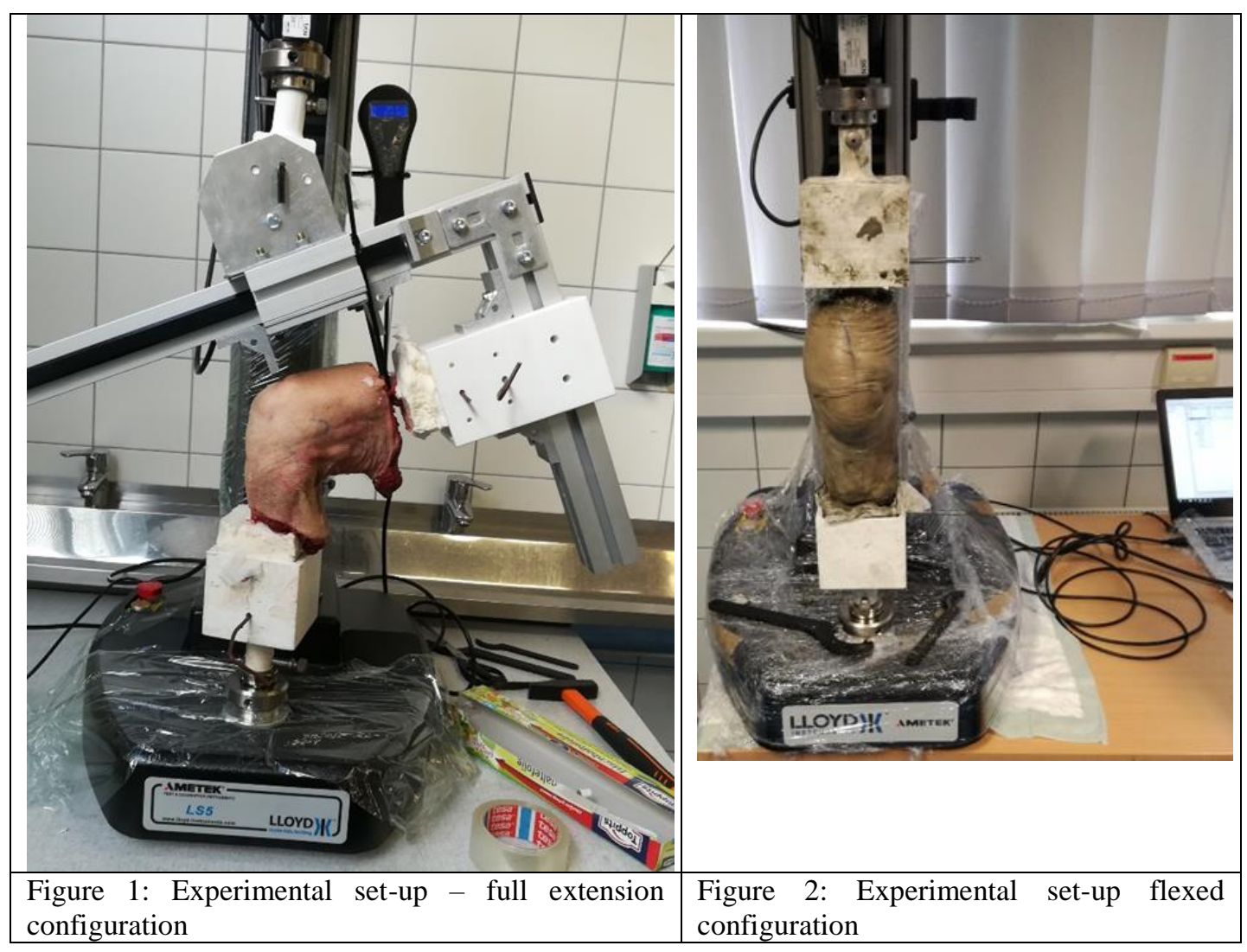

\section{References}

Manning, W., Blain, A., Longstaff, L., \& Deehan, D. (2018). A load-measuring device can achieve fine-tuning of mediolateral load at knee arthroplasty but may lead to a more lax knee state. Knee Surgery, Sports Traumatology, Arthroscopy, 27. 10.1007/s00167-018-5164-3. 